\title{
INSIGHT INTO BASELINE TROUBLES AND PROBLEM RESOLUTIONS IN HIGH-PERFORMANCE LIQUID CHROMATOGRAPHY
}

\author{
ATOLE D*, DEOKATE UA \\ Department of Pharmaceutical Chemistry, Government College of Pharmacy, Aurangabad, Maharashtra, India. \\ Email: dipali@destinationpharmagens.com
}

Received: 04 March 2020, Revised and Accepted: 28 April 2020

\begin{abstract}
For pharmaceutical industry, HPLC is more than 40 years old. Even after instrumental advancements in HPLC problems still arise, troubleshooting still comes. HPLC is the main stay of analytical section nowadays in pharmaceutical industry whether it is API industry or formulation plant. Best approach for troubleshooting HPLC problems is going with systematic way. At the start of quantitative chromatographic analysis, the first parameter of paramount importance is baseline, especially for measuring the area of any given peak. Baseline usually suffers from these errors, namely, high baseline drift, periodic baseline fluctuation, and spikes. This article aims at suggesting some working methodologies which will resolve as well as avoid cases of such errors and failures.
\end{abstract}

Keywords: HPLC, Troubleshooting, Baseline, Pharmaceutical industry.

(C) 2020 The Authors. Published by Innovare Academic Sciences Pvt Ltd. This is an open access article under the CC BY license (http://creativecommons. org/licenses/by/4. 0/) DOI: http://dx.doi.org/10.22159/ajpcr.2020.v13i7.37336

\section{INTRODUCTION}

The practice of HPLC is more than 40 years old in pharmaceutical industry. Although HPLC has been improved by many advances in instrumentation and highly pure chemical components, problems still arise. The active working components of HPLC system are same as before with some upgrades such as pre-column heater of 5-10 $\mu \mathrm{L}$ [1-3].

Validation of analytical methods is important for generating authentic and reliable data in case regulatory submissions which is generated using that method. These methods are essential for a variety of purposes, including testing for QC release, testing of stability samples, and testing of reference materials and to produce data to support specifications [1].

\section{Role of HPLC at present day in Pharmaceutical Industry}

HPLC is the main stay of analytical section nowadays in pharmaceutical industry whether it is API industry or formulation plant. Quality control is an important department of the pharmaceutical industry for commercialization of drugs. In quality control department, spectroscopic instruments are no longer reliable considering 21-CFR and auditing agencies demand. In analysis, at every stage of process, for API or formulation, HPLC is essentially required. Greater advantage with HPLC systems is automation associated with sampling sequence, sampling chamber temperature, column temperature, column choice flexibility considering its length or stationary phase material, and systems capacity to run for long time without any failure make are best tool for routine analysis. So far, as QC department concerned, HPLC instrument is workforce for productivity or output for the pharmaceutical industry [2-32].

To support this giant role of HPLC, we can also overlook on the commercial market of HPLC system and its parts as HPLC market, including supply of columns and other accessories, was of worth $\$ 3.2$ billion. The compounded annual growth for HPLC is forecasted at $6 \% /$ year for the years bringing estimated revenues to nearly $\$ 4$ billion in the past decade till 2010 [21].

Now, what will happen if this automated HPLC system faces problem due to physicochemical property of solution or hardware related problem? Yes, this happens routinely while working. Proper handling of system and making solution accurately can definitely delay such incidences but cannot avoid it.
Consequence of error or failure associated with HPLC system Any kind of error or failure obviously causes incidence or deviation. An incidence is an resultant event of our sop activity that can definitely harm our product quality as well as it is against Cgmp. Incidence is non c-GMP (current good manufacturing practices) event occurred all of sudden and there is uncertainty about its impact on product quality. For example, we have checked column fitting but during mobile phase run due to pressure development, it started leaking and false result obtained in analysis. It will be considered as incidence since we operated as per SOP and still the event occurred. Deviation is defined as when we are deviating from written procedure or GMP (good manufacturing practices) document $[33,34]$.

For HPLC, a more elaborate and sophisticated experimental setup is required. Following are the different parts of HPLC in Fig. 1.

\section{TROUBLESHOOTING}

\section{Need of troubleshooting}

HPLC personnel working at laboratory floor is aware of both facts of incidence and deviation, this article aims at suggesting some working methodologies which will resolve as well as avoid cases of such errors and failures $[35,36]$.

\section{Troubleshooting strategy [35]}

Any troubleshooting strategy involves four steps:

1. Identification of the problem with the help of system indicators or with knowledge of operator

2. Guidance about troubles and troubleshooting to HPLC operator

3. Detection of cause of the trouble

4. Rectification of the causes

Troubleshooting process [35]

1. Gather the facts.

2. Check the simplest things first.

3. Comparison of performances at normal and at trouble.

4. List possible causes.

5. Step-by-step workout on possible causes to check the outcome from changes developed.

6. Call system engineers or service support team 


\section{Baseline}

The portion of the chromatogram recording the detector response when only the mobile phase emerges from the column. In quantitative chromatographic analysis, the baseline is duration of a chromatogram during which only mobile phase is running through the detector and is the reference point for when measuring the area of any given peak [38-43].

To accurately develop analytical methods, to generate data (peaks), for integrating and reporting results, a smooth and flat baseline is always essential. Any signal present on the baseline represents the noise of the chromatographic system (in the absence of injected sample). While continuous deviation from zero absorbance is referred as drift, depending on the direction, it could be positive drift or negative drift as shown in Fig 2. Lower sensitivity and upward baselines, noise, or spike on the chromatogram can often be ascribed to the mobile phase. Apart from mobile phase system components also contribute to baseline errors [38-43].

\section{High baseline drift}

1. Due Non-Homogenous mobile phase

If single solvent is used as mobile phase, and in chromatogram, high baseline drift is observed then most common cause of such baseline drift is the grade of solvent used as mobile phase.

If gradient mobile phase combination is being used and high baseline shift is observed at that time, major responsible factors are different UV cutoff or different absorptivity properties of solvents. In such cases, it is recommended to use baseline subtraction method $[44,45]$.

Apart from this problem arises with gradient program in baseline is due to improper mixing of mobile phase components. Many times industrial pharmaceutical application involves mixture of organic solvents with two or more buffers. Here proper mixing of mobile phase components is important, considering miscibility factor of individual buffer solutions with another one.

\section{Compounds eluting off column}

Many times, the previously performed analysis is the cause of troubles or errors observed in ongoing analysis. Compound constantly eluting is the reason of drift in mobile phase, such kind of drift is detected or diagnosed by multiple wavelength detector or PDA detector of HPLC. Here, it is recommended either to run strong solvent until baseline is stable or suitable organic solvent of previous analysis [47].

\section{High noise}

Noise sometimes has a lot of higher frequency in comparison to chromatographic signal and it is the short period of the baseline from a line caused by electrical signal fluctuations, detector, environmental fluctuations, and different connected factors. Noise is that the issue limits detector sensitivity. Difference between noise and drift is presented in Fig. 3. For this noise, quantitative detection limit kept as 10x signal to noise. This ensures accurate determination of the amounts with less than $2 \%$ difference. Noise is often measured peak to peak, i.e., the space from the highest of 1 such little peak to the lowest of following. Sometimes, noise is averaged over a nominal amount of your time [48].

There are two types of noise such as;

Synchronous noise: It is a periodic noise usually associated with pump Strokes as shown in Fig. 4 [29,49,50].

Asynchronous noise - It is a random noise usually associated with contaminated mobile phases or its components, also due to its poor mixing; dirt on detector cell, leak at detector cell; degraded samples, impurities in samples or solvents $[29,50]$.

Most common cause of these both noises in baseline is dirt in the detector cell or detector window. This can be solved by flushing detector cell with high flow rates using suitable organic solvent. In baseline, random appearance of noise and spikes occurs, variation in flow rates and/or pressures observed, detector outputs to show high

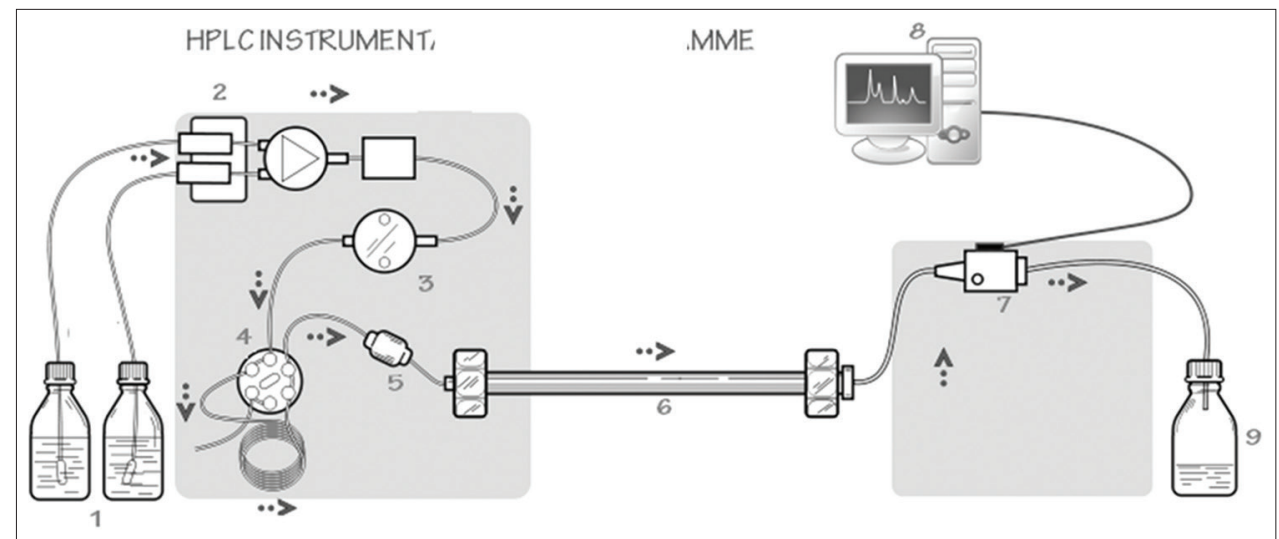

Fig. 1: HPLC schematic diagram. 1: Mobile phase reservoir, 2: Pump, 3: Mixing chamber, 4: Chamber, 5: Guard column, 6: Analytical column, 7: Detector, 8: Monitor and recording system, 9: Outlet reservoir

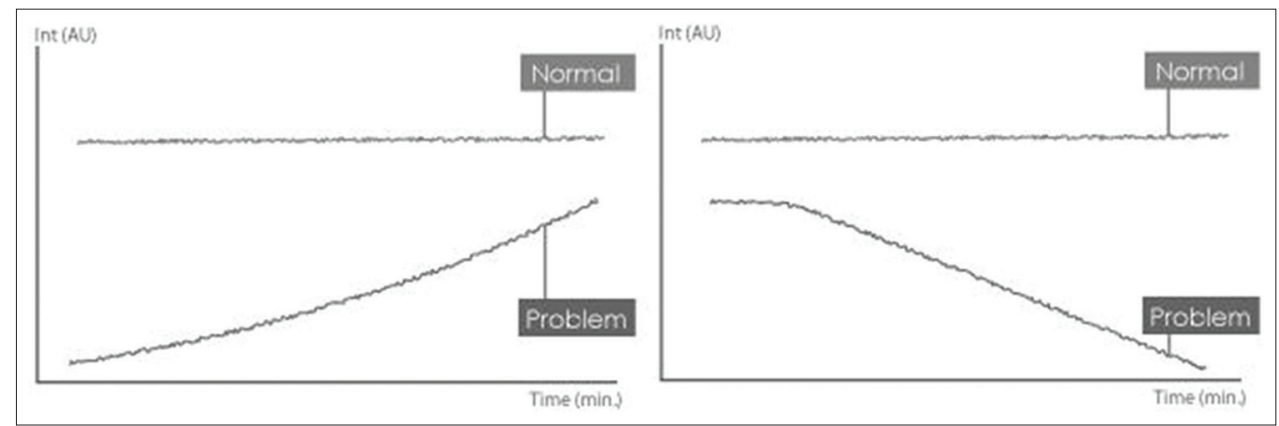

Fig. 2: Positive (left) and negative (right) baseline drift [37] 
levels of noise. To avoid this, mobile phases should be fully degassed before use [51].

Apart from this, other factors contributing baseline noise are temperature fluctuations in the mobile phase mixture during the chromatographic run. If detector cell is clean, mobile phase is properly degassed and temperature is well maintained then baseline noise presented in Fig. 5 is indicating lamp failure [52,53].

\section{Periodic baseline fluctuation}

1. Pressure fluctuations from pump

Common causes of this pressure fluctuation associated with system hardware are: HPLC back pressure too low to maintain prime in system, worn or damaged pump piston seals, leaking or worn pump piston seal, and improper fitting of column or inline tubing. Such problems can be resolved by proper training and technique to use system, but most importantly, it is maintenance related so it is advised to keep system in excellent condition. Also, perform regularly preventative maintenance and schedule inspection and cleaning of entire system to eliminate unproductive down time [54].

Common causes of this pressure fluctuation associated with liquid component used in mobile phase are as follows: Flow rate inappropriate in the gradient program, mixing problem (gradient or isocratic online

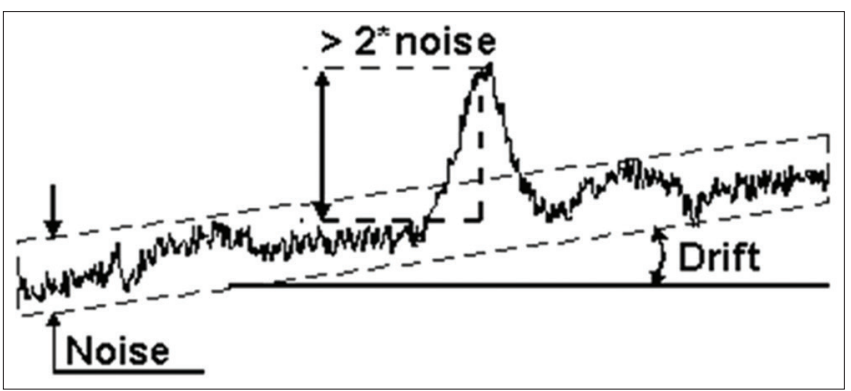

Fig. 3: Baselines, noise on the chromatogram

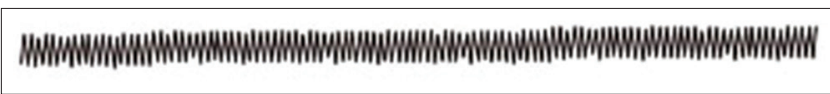

Fig. 4: Synchronous noise

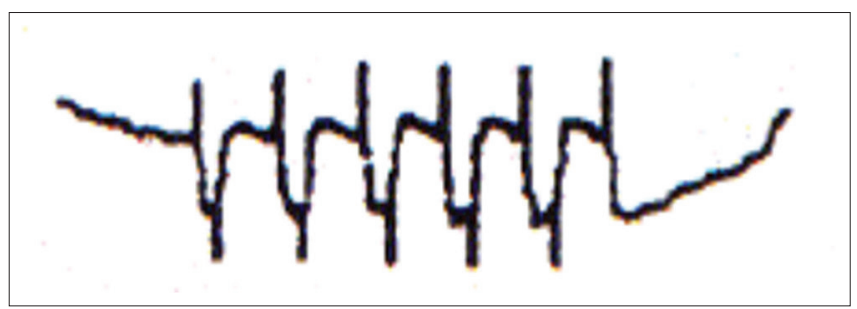

Fig. 5: Noise due to lamp failure [51]

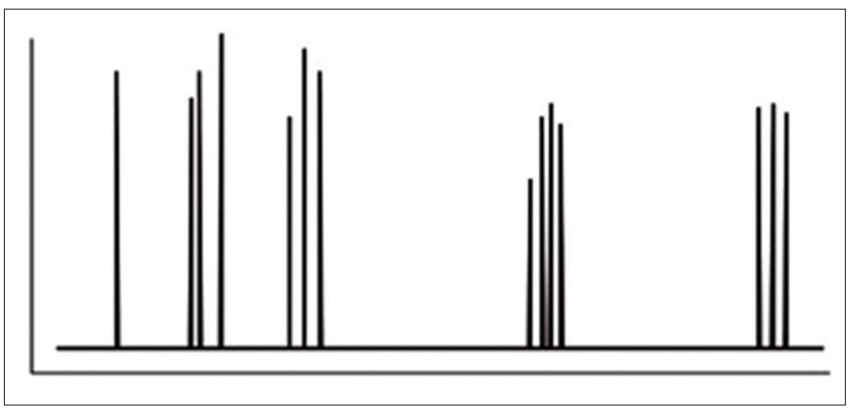

Fig. 6: Spike in chromatograms mixing), bubble in the tubing at any location, sticking or old or unchanged check valve solution, and poor mobile phase miscibility. Such problems can be resolved by degasing mobile phase, best with on-line vacuum degassing or helium sparging. Purge the pump. Actively draw liquid through the pump, for instance with a syringe at the outlet of the pump $[55,56]$.

Additional causes of this pressure fluctuation include insufficient mixing of mobile phase solution and degraded solution of mobile phase and wrong reference wavelength selected solution.

\section{Spikes}

1. Spikes Due to electrical interferences

Spikes are electrical signals, are initiated by an external event. Spikes are typically not random, but for instance related to cyclic strong power consuming equipment as shown in Fig 6. Isolate the electrical supply to filter current fluctuations, so as to avoid spikes in the chromatogram due to this reason [31].

\section{Lamp relay trying to fire a dead lamp}

Detector-related problems include air bubbles in the tubing, and optical cell contamination or interferences. These usually produce spikes on the chromatograms or low sensitivity [31-58].

If not possible to resolve with in house personnel, the best option is to communicate a service engineer. These spikes could also be due to air bubbles in the detector cell, less likely with the new generation of inline vacuum degassing systems $[50,58]$.

\section{CONCLUSION}

Hence, as far as pharmaceutical including overall departments, HPLC instrument is workforce for productivity or output for the pharmaceutical industry whether it is API industry or formulation plant. With the many advances in instrumentation and highly pure chemical components, but problems still arise usually due to grade of solvent for mobile phase combination or their improper mixing, previously retained compound, electricity fluctuations, dirt in the detector cell, or detector window. Without systematic approach identification of problem for troubleshooting, HPLC system is not possible. At the start of quantitative chromatographic analysis, first parameter of paramount importance is baseline, especially for measuring the area of any given peak. Baseline usually suffers from these errors, namely, high baseline drift, periodic baseline fluctuation, and spikes. Best option considering productivity or output will be to avoid generation of problems which can be achieved by scheduled maintenance activities by service engineers, use of high-quality HPLC grade chemicals and reagents, and finally, most important operating instrument under stated standard operating conditions. Mobile phase use should be used with proper care will sure generate proper generate without need of troubleshooting.

\section{CONFLICTS OF INTEREST}

The authors mentioned certify that they have no affiliations with or involvement in any organization or entity with any financial interest or non-financial interest in the subject matter or materials discussed in this manuscript.

\section{AUTHORS' FUNDING}

We did not receive any funding for the present review.

\section{REFERENCES}

1. International Conference on Harmonization of Technical Requirements for Registration of Pharmaceuticals for Human Use. Validation of Analytical Procedures: Text and Methodology (Q2R1) Geneva, Switzerland: International Conference on Harmonization of Technical Requirements for Registration of Pharmaceuticals for Human Use; 2005.

2. Bridwell H, Dhingra V, Peckman D, Roark J, Lehman T. Perspectives on method validation: Importance of adequate method validation. Qual 
Assur J 2010;13:72-7.

3. Gorog S. The changing face of pharmaceutical analysis. Trends Anal Chem 2007;26:12-7.

4. Scott PW. Liquid Chromatography for the Analyst. New York: Marcel Dekker Inc.;1994. p. 1-10.

5. Stanley BJ, Foster CR, Guiochon G. On the reproducibility of column performance in liquid chromatography and the role of the packing density. J Chromatogr A 1997;761:41-51.

6. Christain GD. Analytical Chemistry. $6^{\text {th }}$ ed. United States: John Wiley \& Sons Inc.; 2001.

7. Nledner W, Karsten M, Stelner F, Swart R. Automating Method Development with an HPLC System Optimized for Scouting of Columns, Eluents and Other Method Parameters. United States; Pittcon Presentation; 2008.

8. Kar A. Pharmaceutical Drug Analysis. 1st ed. Tamil Nadu: New Age International Private Limited; 2001. p. 565-92.

9. Skoog DA, West DM, Holler FJ, Crouch SR. Fundamentals of Analytical Chemistry. $8^{\text {th }}$ ed. Tamil Nadu: Brooks/Cole; 2004. p. 973.

10. Scott RP. Principles and Practice of Chromatography (Chrom-Ed Book Series). New York: Reese-Scott Partnership; 2003. p. 1-2.

11. Chatwal GR, Anand SK. Instrumental Methods of Chemical Analysis. $5^{\text {th }}$ ed. Uttar Pradesh: Krishna Prakashan Media (P) Ltd.; 2004.

12. U.S. Food and Drug Administration. Title 21 Code of Federal Regulations (21 CFR Part 211.194(a)(2)). Laboratory Records. Available from: http://www.accessdata.fda.gov/scripts/cdrh/cfdocs/ cfcfr/CFRSearch.cfm?fr=211.194. [Last accessed on 2009 Dec 11].

13. Chervet JP, Ursem M, Salzmann JP. Instrumental requirements for nano-scale liquid chromatography. Anal Chem 1996;68:1507-12.

14. Saito Y, Jinno K, Greibrokk T. Capillary columns in liquid chromatography: Between conventional columns and microchips. J Sep Sci 2004;27:1379-90.

15. Hernandez-Borges JH, Aturki Z, Rocco A, Fanali S. Recent applications in nano-liquid chromatography. J Sep Sci 2007;30:1589-610.

16. Qing H. Integrated Nano Liquid Chromatography System on a Chip, Ph.D. Thesis. California, USA: California Institute of Technology Pasadena; 2005

17. Tzanavaras PD, Themelis DG. Review of recent applications of flow injection spectrophotometry to pharmaceutical analysis. Anal Chim Acta 2007;588:1-9.

18. Hassan BA. HPLC uses and importance in the pharmaceutical analysis and industrial field. Pharm Anal Acta 2012;3:9.

19. Gumustas M, Kurbanoglu S, Uslu B, Ozkan S. UPLC versus HPLC on drug analysis: Advantageous, applications and their validation parameters. Chromatographia 2013;76:21-2.

20. Glajch JL. Impact of new columns on drug development. Chromatogr Online 2014;32:36-41.

21. Thammana M. A review on high-performance liquid chromatography. Res Rev 2016;5:22.

22. Malviya R, Bansal V, Pal OP, Sharma P. High-performance liquid chromatography: A short review. J Glob Pharm Technol 2010;2:22-6.

23. Bokai M, Xin-Lei G, Xin-Ying Z. Application of high performance liquid chromatography in food and drug safety analysis. J Food Saf Qual 2016;7:4295-8

24. Salgado P, Visnevschi-Necrasov T, Kiene RP, Azevedo I, Rocha AC, Almeida CM, et al. Determination of 3-mercaptopropionic acid by HPLC: A sensitive method for environmental applications. J Chromatogr B 2015;992:103-8.

25. Bird IM. High-performance liquid chromatography: Principles and clinical applications. Br Med J 1989;299:783-7.

26. Rogatsky E. Modern high performance liquid chromatography and HPLC 2016 international symposium. J Chromatogr Sep Tech 2016;7:135

27. Wu N, Clausen AM. Fundamental and practical aspects of ultrahigh pressure liquid chromatography for fast separations. J Sep Sci 2007;30:1167.

28. Kirkland JJ. Modern Practice of Liquid Chromatography. New York: Wiley-Inter Science; 1971

29. Crowther JB, Lauwers W, Adusumalli S, Shenbagamurthi P. The laboratory analyst's role in the drug development process. In: Miller JM, Crowther JB, editors. Analytical Chemistry in a GMP Environment. A Practical Guide. New York: John Wiley; 2000. p. 448-9.

30. Dong MW. Modern HPLC for Practicing Scientists. Ch. 5. United States: John Wiley \& Sons Publication; 2006. p. 111-6.

31. Prathap B, Deyb A, Raoa GH, Johnsona P, Arthanariswaranc P. A review-importance of RP-HPLC in analytical method development. Int J Novel Trend Pharm Sci 2013;3:15-23.

32. Available from: https://www.biospectrumindia.com/news/73/2472/ hplc-market-in-india-growing.html.

33. Available from: https://www.who.int/immunization_standards/ vaccine quality/risk july 2013.

34. Available from: https://www.pharmamanual.com/deviation-andincidence-difference.

35. Academy Savant. Troubleshooting in HPLC, CLC-70, (Computerbased Instruction). Fullerton, CA: Academy Savant; 2020

36. McNaught AD, Wilkinson A. Compendium of Chemical Terminology. $2^{\text {nd }}$ ed. Oxford: Blackwell Scientific Publications, IUPAC; 1997.

37. Available from: https://www.chromacademy.com/chromatographyHPLC-Gradient-Elution-Baseline-Drift.html

38. Available from: https://www.chromservis.eu/c/hplc-troubleshooting.

39. Available from: http://www.ucd.ie/t4cms/VWR $\% 20 H P L C \% 20$ Troubleshooting.pdf.

40. Available from: https://www.sigmaaldrich.com/content/dam/sigmaaldrich/docs/Supelco/Bulletin/4497.pdf.

41. Available from: https://www.chromacademy.com/hplc-troubleshootingguide-cycling-baselines-pressure-fluctuations.html.

42. Available from: https://www.agilent.com/cs/library/posters/public/ Final $\% 20$ TIPS $\% 20$ and $\% 20$ Tricks $\% 20$ HPLC $\% 20$ Troubleshooting $\% 20$ (2).pdf.

43. Available from: https://www.phenomenex.blob.core.windows.net/ documents/a8cb7bc3-eb65-4244-8910-faeb1818df8c.pdf.

44. Available from: http://www.kb.mtc-usa.com/article/AA-01273/0/ATip-to-improve-or-obtain-flat-baselines-in-HPLC-UV-gradientapplications.html.

45. Filgueiraa MR, Castells CB, Carra PW. A simple robust orthogonal background correction method for two-dimensional liquid chromatography. Anal Chem 2012:84;6747-52.

46. Snyder LR, Kirkland JJ, Dolan JW. Introduction to Modern Liquid Chromatography. United States: Wiley; 2009

47. Neue UD, Corperation W. HPLC Troublr Shooting Guide. Available from: Waters [Last accessed 2020 Feb 20].

48. Available from: http://www.hplc.chem.shu.edu/NEW/HPLC Book/ Detectors/det_nise.html.

49. Available from: http://www.chromedia.org/chromedia?waxtrapp=lxsaq EsHiemBpdmBlIEcChBgC\&subNav=pmxgzFsHiemBpdmBlIEcCh $\mathrm{BgCdD}$.

50. Mcnair H, Polite LN. Troubleshooting in high performance liquid chromatography. Sep Sci Technol 2007;8:459-77.

51. Nelson MD, Wolan JD. UV detector noise. LC GC 1999;17:12-5

52. Available from: http://www.hplctips.blogspot.com/2014/01/ diagnosing-troubleshooting-hplc.html.

53. Available from: http://www.hplc.chem.shu.edu/NEW/HPLC_Book/ Detectors/det nise.html.

54. Available from: https://www.hplctips.blogspot.com/2014/01/ diagnosing-troubleshooting-hplc.html.

55. Burghate AV. A review on HPLC-trouble shooting guide. Int J Pharm Sci Rev 2014;27:200-9.

56. Snyder LR, Kirkland JJ, Glajch JL. Practical HPLC Method Development. $2^{\text {nd }}$ ed. New York: Wiley-Inter Science; 1997.

57. Wolan JD. LC user survey: Detector problem. LC GC1986;4:526-9.

58. Sadek PC. Troubleshooting HPLC Systems: A Bench Manual. New York: John Wiley \& Sons; 2000. 